\title{
Holmes" Tremor in an HIV Positive Patient Worsened by Immune Recovery Inflammatory Syndrome (IRIS)
}

\author{
Rosalie B. Corrêa', Felipe R. Schmidt', Fernanda MLC Silva ${ }^{1}$, Flavio Henrique R. Costa ${ }^{1}$, Ana Lucia Rosso ${ }^{1 *}$, James P. de Mattos ${ }^{1}$, Denise H. \\ Nicaretta ${ }^{2}$ and Sergio A.P. Novis ${ }^{1,2}$
}

${ }^{1}$ Serviço de Neurologia Prof. Sergio Novis, Hospital Universitário Clementino Fraga Filho (UFRJ), Rio de Janeiro, Brazil ${ }^{2} 25^{a}$ Enfermaria da Santa Casa de Misericórdia do Rio de Janeiro, Brazil

IRIS is characterized by a paradoxical deterioration of clinical status after initiation of Anti-Retroviral Therapy (ART), despite improved immune function. It is caused by inflammatory response against the infectious antigen [1]. IRIS typically occurs in patients with a low initial CD4 (usually $<50$ ) and a rapid decline in viral load [2]. It is seen within a broad spectrum of HIV-related opportunistic infectious diseases and autoimmune disorders in patients who had been given Highly Active Anti-Retroviral Therapy (HAART) [3]. Our objective is to describe an HIV positive patient with Holmes' tremor worsened by IRIS, with marked recovery after therapy with steroids.

A 41-year-old woman presented with esophageal candidiasis and then she was found to be positive for HIV. A month later she complained of headache and tremors. Upon examination she had a mild rest, postural and action tremor, which was more pronounced on the left side. There was a discretely ataxic gait, pyramidal signs and hypoesthesia on the left side, multidirectional nystagmus, and right abducens and bilateral peripheral facial palsy. She had a CD4 count of $32 \mathrm{cells} / \mathrm{mm}^{3}$ and a viral load of $>500,000$ copies $/ \mathrm{ml}$. Brain MRI showed a hyperintense signal on T2 and Flair, extending from the medulla oblongata to the right thalamus, with no contrast enhancement. Her CSF was normal and negative for anti-JC virus. She was treated initially with stavudine, lamivudine and lopinavir/ritonavir. Three months later the tremor had worsened, and she was unable to walk without assistance or stand upright with her eyes open, because of the great intensity of the head tremor. Subsequently, she developed tetraparesis and excessive somnolence. The CD4 count was 264 cells/ $\mathrm{mm}^{3}$ and the viral load 38,837 copies/ml. Brain MRI showed multiple scattered bilateral subcortical lesions with high-intensity signal in T2 and Flair. The prior lesion had grown in volume. Brain biopsy failed to reveal features of Progressive multifocal leucoencephalopathy (PML) or lymphoma, but scattered positive CD8 cells were observed. Oral prednisone $60 \mathrm{mg} / \mathrm{day}$, topiramate $200 \mathrm{mg} / \mathrm{day}$ and clonazepam 1.5 $\mathrm{mg} /$ day were started, with moderate improvement of the symptoms. The combination of lopinavir/ritonavir was switched to efavirenz. One year after being diagnosed with HIV, the patient presents only moderate postural and action tremor in the left arm.

The term IRIS has been used to describe two clinical conditions: the paradoxical worsening of pre-existent opportunistic infection or the unmasking of a subclinical opportunistic infection after the initiation of ART [2]. Although most patients present the second condition, the neurological manifestations in our patient occurred before starting ART. The clinical manifestations of IRIS include high fever, lympho node enlargement, respiratory distress, meningitis, palsies, paresthesias, behavioral disorders, headache, tremor and ataxia [3]. It usually appears within the first 4-12 weeks after starting ART. Our patient's neurological symptoms worsened 12 weeks after starting ART, manifested by intense rest, postural, and action tremor which made it hard for her to open her eyes and sit up in bed. These clinical features are described in brainstem lesions (Holmes' tremor). In the present case, a similar lesion is found in the MRI images, extending from the medulla oblongata to the thalamus. There are two possible etiologies for this lesion: PML or HIV leucoencephalopathy. In our patient, the MRI initially showed a non-enhancing, demyelinating lesion with no mass effect, in the brainstem. After a few weeks, the lesion had extended to the cerebral cortex and had developed an inflammatory reaction, with contrast enhancement and a mass effect (Figure 1), suggesting IRIS-related PML. Because the brain biopsy and CSF exam were inconclusive for PML, HIV encephalopathy could not be completely ruled out. The diagnosis of IRIS is based on the exclusion of other causes such as worsening of opportunistic infection due to failure of treatment, onset of a new opportunistic infection and drug toxicity [2]. Many open studies have indicated the efficacy of corticosteroids in the treatment of IRIS, but the exact doses and duration of the treatment have not yet been established [3]. Corticosteroids should be recommended for patients with severe and progressive symptoms, whose MRI demonstrates inflammatory lesions.

In conclusion, to the best of our knowledge this is the first report of an HIV-positive patient with Holmes' tremor aggravated by IRIS, probably secondary to PML.

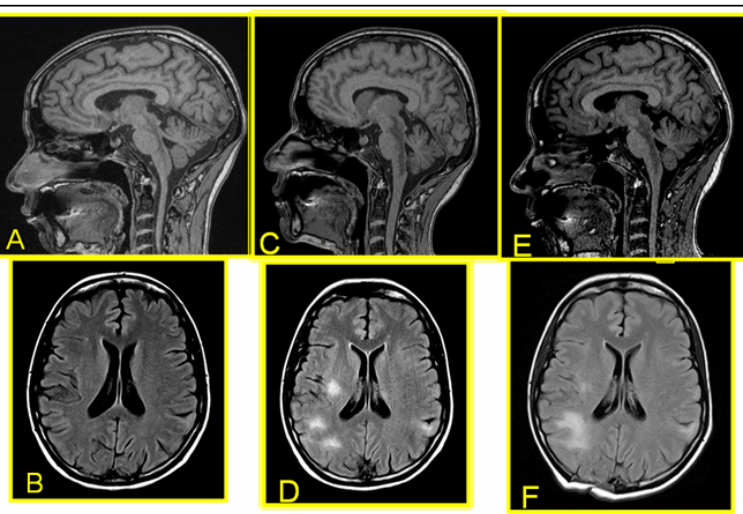

Figure 1: Before ART: (A) sagittal T1 imaging showing a posterior low signal intensity lesion extended from medulla oblongata to diencephalus; (B) axial Flair without subcortical lesions. Weeks after ART: (C) sagittal T1 imaging with enlargement of the lesion; (D) axial Flair showing high intensity lesions scattered in the subcortical areas. Months after ART: (E) sagittal T1 and (F) axial Flair images with mild improvement.

*Corresponding author: Dr. Ana Lucia Rosso, Serviço de Neurologia Prof. Sergio Novis, Hospital Universitário Clementino Fraga Filho (UFRJ), Rua Santa Clara 50 702, Rio de Janeiro, RJ, Zip: 22041-012, Brazil, Fax: +55-21-25622738; E-mail: anarosso@gmail.com

Received October 13, 2010; Accepted November 03, 2010; Published November 08,2010

Citation: Corrêa RB, Schmidt FR, Silva MLCF, Costa FHR, Rosso AL, et al. (2010) Holmes' Tremor in an HIV Positive Patient Worsened by Immune Recovery Infl ammatory Syndrome (IRIS). J AIDS Clinic Res 1:105. doi:10.4172/2155-6113.1000105

Copyright: (c) 2010 Corrêa RB, et al. This is an open-access article distributed under the terms of the Creative Commons Attribution License, which permits unrestricted use, distribution, and reproduction in any medium, provided the original author and source are credited. 
Citation: Corrêa RB, Schmidt FR, Silva MLCF, Costa FHR, Rosso AL, et al. (2010) Holmes' Tremor in an HIV Positive Patient Worsened by Immune Recovery InflammatorySyndrome(IRIS).JAIDSClinicRes 1:105.

doi:10.4172/2155-6113.1000105

Page 2 of 2

\section{Acknowledgments}

We thank Prof. Eric Sweet for his kind assistance in reviewing the manuscript.

\section{References}

1. Page KR, Andrade A Immune reconstitution inflammatory syndrome (IRIS).
2. Beishuizen SJ, Geerlings SE (2009) Immune reconstitution inflammatory syndrome: immunopathogenesis, risk factors, diagnosis, treatment and prevention. Neth J Med 67: 327-331.

3. Kaplan JE, Benson C, Holmes KH, Brooks JT, Pau A, et al. (2009) Guidelines for Prevention and Treatment of Opportunistic Infections in HIV-Infected Adults and Adolescents. MMWR 58: 1-207. 